\title{
Epuration chimique et stérilisation microbiologique par traitement thermique des saumures utilisées dans l'industrie alimentaire et en particulier dans l'industrie fromagère ${ }^{(1)}$
}

\author{
par \\ M. VIARD et J. DEVEAU \\ Laboratoire de Recherche de la \\ Compagnie des Salins du Midi et des Salines de l'Est
}

Le procédé de salage des fromages par saumurage consiste généralement à immerger le produit à traiter dans une saumure ayant une concentration fixe en chlorure de sodium durant un temps donné et à une température déterminée. On sait que, au cours du séjour des fromages dans cette saumure, il se produit un échange osmotique qui a pour résultat :

- d'une part, une diminution de la concentration en sel résultant de sa pénétration dans le produit à traiter et de la dilution de la saumure par l'eau résultant de l'échange osmotique,

- d'autre part, une augmentation de la teneur en impuretés de cette saumure.

C'est ainsi que des impuretés minérales sont libérées par le fromage et sont apportées par le sel ou par l'eau de dissolution. De même, des impuretés organiques (lactose - acide lactique, etc.) sont libérées par le fromage.

Enfin, il se produit un enrichissement bactérien et fongique de la saumure ; cet effet est variable suivant le type de fromage traité.

En ce qui concerne les pâtes molles moisies, des études ont été réalisées à l'Ecole Supérieure de Laiterie de Nancy [2]. Les résultats montrent qu'au cours du saumurage de fromages type camembert, il se produit une augmentation en microcoques pigmentés et surtout non pigmentés, en coliformes (Escherichia coli), en moisissures (Aspergillus, Penicillium glaucum Link, Mucor globosus, Penicillium candidum Link ou caseicolum Bainier que l'on retrouve après ensemencement dans le lait lors de la fabrication des pâtes molles), en levures (Saccharomyces et Torula).

Dans ces conditions, même si l'on maintient à une valeur relativement basse la température des saumures, on constate un enrichis-

(1) Cette étude a fait l'objet du dépôt du brevet $\mathrm{n}^{\circ}$ 7039-677. 
sement très rapide de la flore bactérienne et fongique, ce qui se traduit, en premier lieu, par une mauvaise odeur et un mauvais goût et ensuite par une altération du fromage. Ces inconvénients résultent de la dégradation des substances fermentescibles apportées à la saumure par le fromage. Enfin, s'y ajoutent les accidents de flore provoqués par un ensemencement de levures et de champignons indésirables.

En raison de cette dégradation de la saumure résultant de l'augmentation du taux des impuretés, on est, en général, conduit à purger fréquemment une certaine quantité, voire la totalité, de la saumure utilisée. Ce processus, indépendamment de son incidence sur le prix de revient du saumurage, n'est pas satisfaisant techniquement car il modifie constamment les caractéristiques chimiques et bactériologiques de la saumure utilisée.

Afin de remédier à ces inconvénients, il est connu que l'on peut stériliser les saumures en continu par le procédé classique de pasteurisation [6 et 7] mais, dans la pratique, ce procédé entraîne des inconvénients et des difficultés dus à l'entartrage des surfaces de chauffe par les sulfates et les carbonates présents dans la saumure.

On peut également chercher à obtenir cette stérilisation par l'action des rayons ultra-violets [1], mais ce procédé a l'inconvénient de ne pas réaliser en même temps l'épuration chimique des saumures (élimination par précipitation des sulfates et des carbonates).

Enfin, on peut également obtenir une certaine stérilisation en ajoutant aux saumures des antiseptiques comme l'eau oxygénée [5] ou l'hypochlorite de soude [4] mais cette opération est délicate et elle ne réalise pas non plus l'épuration chimique.

Les travaux entrepris ont permis d'aboutir à un procédé qui réalise simultanément la stérilisation en continu des micro-organismes contenus dans la saumure en circulation et l'épuration chimique de celle-ci, tout en évitant les inconvénients et les difficultés mentionnées ci-dessus. Ce procédé est évidemment applicable non seulement aux saumures de fromagerie, mais aussi aux saumures utilisées dans l'industrie alimentaire.

Ce procédé consiste à chauffer la saumure jusqu'à une température égale ou supérieure à $100^{\circ} \mathrm{C}$ durant $45 \mathrm{mn}$, en présence de micro-cristaux de carbonate de chaux et de sulfate de chaux. Ce temps de chauffage doit être considéré comme un temps maximum. Un chauffage à $104^{\circ} \mathrm{C}$ pendant $20 \mathrm{mn}$ devrait donner des résultats comparables.

Ces cristaux-germes, dont la granulométrie doit être inférieure à $75 \mu$, sont introduits dans le stérilisateur, en proportion supérieure à $5 \mathrm{~g}$ par litre environ. Du fait de cet ensemencement, les bicarbonates présents dans la saumure sont décomposés et précipitent à l'état de carbonate de calcium en formant un enrobage sur les germes cristallins qui ont été ajoutés. Il en est de même en ce qui 
concerne le sulfate de calcium présent dans la saumure. Quant aux sels de calcium dérivés de l'acide lactique, ils précipitent et restent en suspension. Enfin, les substances protéiques, solubles à froid, floculent.

La saumure ainsi traitée est stérilisée, filtrée puis refroidie avant utilisation.

A titre d'exemple, nous avons rassemblé dans le tableau 1 les résultats analytiques obtenus en marche continue.

TABLEAU 1

Composition de la saumure avant et après traitement

\begin{tabular}{|c|c|c|c|}
\hline \multicolumn{2}{|c|}{ Saumure avant traitement } & \multicolumn{2}{|c|}{ Saumure après traitement } \\
\hline $\begin{array}{l}\text { Insolubles en suspension } \\
\mathrm{Ca} \\
\mathrm{Mg} \\
\mathrm{SO}_{4} \\
\mathrm{Cl} \\
\mathrm{P} \\
\mathrm{pH} \\
\text { Degré Dornic }\end{array}$ & $\begin{array}{rl}1,55 & \mathrm{~g} / 1 \\
1,18 \mathrm{~g} / 1 \\
0,09 \mathrm{~g} / 1 \\
0,28 \mathrm{~g} / 1 \\
162,60 \mathrm{~g} / 1 \\
0,74 \mathrm{~g} / 1 \\
5,10 \\
11\end{array}$ & $\begin{array}{l}\text { Insolubles précipités } \\
\mathrm{Ca} \\
\mathrm{Mg} \\
\mathrm{SO}_{4} \\
\mathrm{Cl} \\
\mathrm{P} \\
\mathrm{pH} \\
\text { Degré Dornic }\end{array}$ & $\begin{array}{cc}4,3 & \mathrm{~g} / 1 \\
0,5 & \mathrm{~g} / 1 \\
0,04 & \mathrm{~g} / 1 \\
0,18 & \mathrm{~g} / 1 \\
162,7 & \mathrm{~g} / 1 \\
0,35 & \mathrm{~g} / 1 \\
4,2 & \\
26 & \end{array}$ \\
\hline
\end{tabular}

L'analyse du précipité obtenu montre, qu'au point de vue minéral, il est essentiellement composé de carbonate de calcium, de phosphate de calcium et de sulfate de calcium et que, du point de vue organique, les constituants sont des matières protéiques. De plus, on constate l'abaissement du $\mathrm{pH}$ de la saumure, lequel atteint une valeur optimale pour le développement des moisissures utilisées en fromagerie. Ceci constitue un résultat important du procédé.

Parallèlement, nous avons contrôlé du point de vue bactériologique des saumures de fromagerie avant et après traitement thermique. Ces saumures considérées proviennent de laiteries de l'Est, leurs compositions microbiologiques sont consignées dans le tableau 2. Il est à noter qu'aucune des saumures analysées ne contient de germes thermorésistants (Bacillus, Clostridium) [3] sous forme sporulée et végétative, néanmoins le traitement thermique appliqué ( $\left.100^{\circ} \mathrm{C}-45 \mathrm{mn}\right)$ semble suffisant pour détruire les formes végétatives.

Les analyses chimiques et microbiologiques de la saumure, avant et après traitement, montrent donc que par simple traitement thermique il est possible d'épurer chimiquement une saumure tout en la stérilisant, sans entartrage des surfaces de chauffe.

L'application pratique de ce procédé peut se réaliser au sein d'une installation de saumurage dont le schéma est représenté sur la figure 1 . 


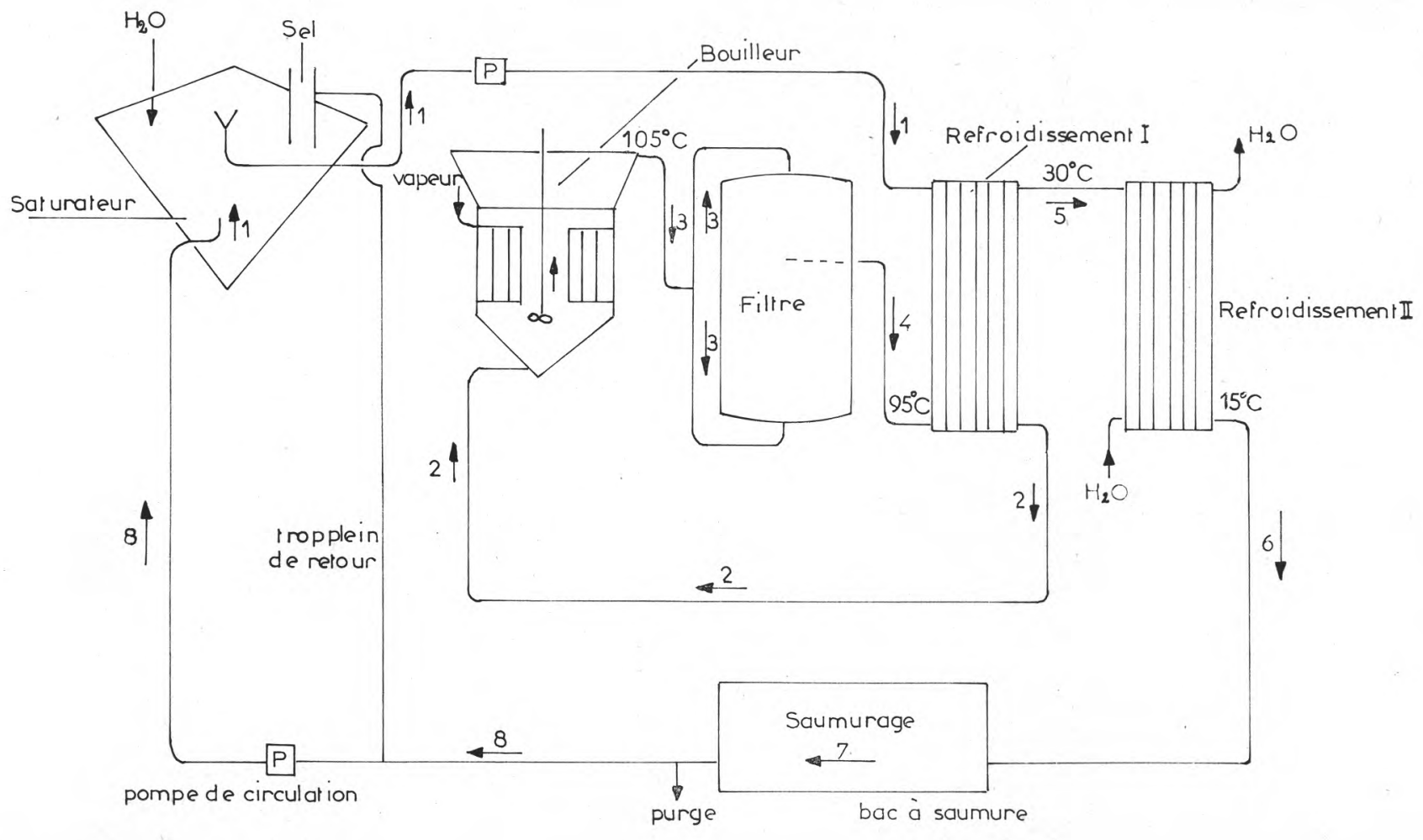

INSTALLATION DE SAUMURAGE POUR EPURERET STERILISER 
Le principe de fonctionnement est alors le suivant : la saumure sort du bac à saumure pour être envoyée dans un saturateur. De là, elle gagne le "bouilleur » en passant préalablement dans un préchauffeur.

Le traitement thermique terminé, la saumure est filtrée, refroidie à $15^{\circ} \mathrm{C}$ et retourne au bac à saumure.

La saumure arrive par la base du " bouilleur » qui est constitué de deux parties (fig. 2) :

- une partie inférieure où la saumure est maintenue en agitation et est chauffée,

- une partie supérieure ou décanteur.

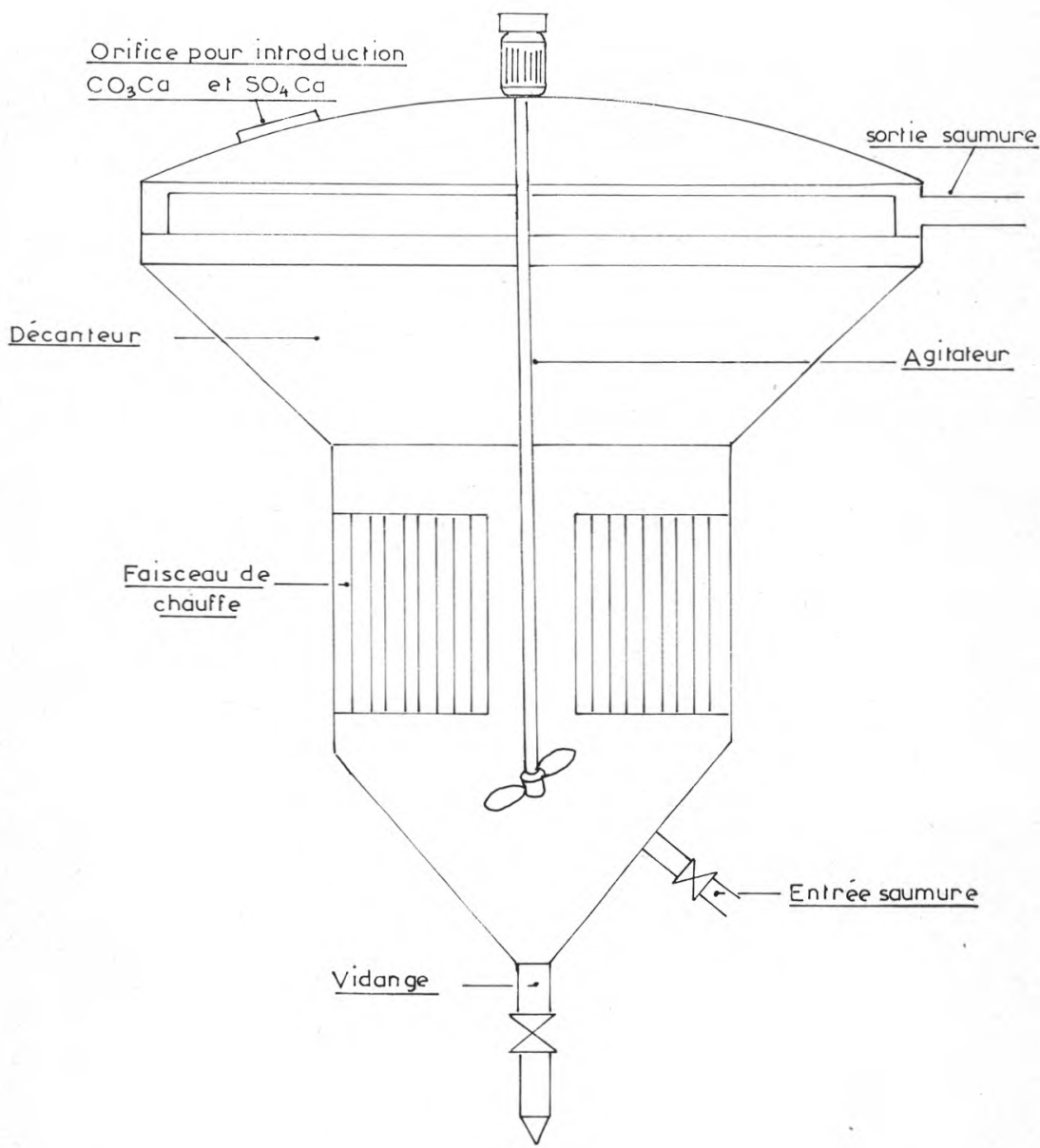

fig. 2 
Il est à noter également que pour des raisons de rendement thermique d'une part et facilité de filtration d'autre part, la saumure est filtrée à chaud.

\section{TABLEAU 2}

Composition microbiologique de la saumure avant et après traitement (par $\mathrm{ml}$ de saumure)

\begin{tabular}{|c|c|c|}
\hline Flore & $\begin{array}{l}\text { Saumure en circulation } \\
\text { avant stérilisation }\end{array}$ & $\begin{array}{l}\text { Saumure traitée à } \\
105^{\circ} \mathrm{C} \text { durant } 45 \mathrm{mn}\end{array}$ \\
\hline $\begin{array}{l}\text { Flore aérobie - mésophile } \\
\text { Bactéries coliformes } \\
\text { Microcoques } \\
\text { Levures } \\
\text { Moisissures }\end{array}$ & $\begin{array}{r}180000 \\
+ \\
8000 \\
100 \\
14000\end{array}$ & $\begin{array}{l}\frac{0}{0} \\
0 \\
0\end{array}$ \\
\hline
\end{tabular}

\section{Ré $\mathbf{s}$ u $\mathrm{m}$ é}

Les impuretés chimiques apportées aux saumures de fromagerie ont triple origine : le sel, l'eau de dissolution, le fromage.

On constate d'autre part un enrichissement bactérien et fongique des saumures au cours de leur vieillissement.

Pour pallier ces inconvénients on a procédé soit à la pasteurisation classique des saumures, soit à une stérilisation par traitement aux rayons ultra-violets, soit à une stérilisation par adjonction de produits antiseptiques.

Aucun de ces procédés n'a permis d'aboutir à des résultats totalement satisfaisants et aucun de ces traitements n'a permis notamment de réaliser conjointement stérilisation et épuration chimique d'une saumure. Un chauffage de la saumure à une température égale ou supérieure à $100^{\circ} \mathrm{C}$, pendant 45 à $60 \mathrm{mn}$, permet en présence de germes de carbonate de chaux et de sulfate de chaux de procéder simultanément à la stérilisation et à l'épuration chimique des saumures, sans entartrage des surfaces de chauffe.

\section{S u m m a ry}

Chemical impurities provided by cheese-dairy brines originate from three facts : salt, dissolving water and cheese.

On an other hand, a bacterial and fungic enrichment can be noted on brines in process of ageing.

So as to palliate these difficulties, it has been proceeded, either to a standard pasteurization of brines, or to a sterilization by means 
of an ultra-violet treatment, or to a sterilization by adding of antiseptic produces.

Of all these processes, not one enabled to result in entirely satisfying effects and fol all these treatment, note one allowed in particular to carry out effect in an interdependant way, sterilization and chemical purification of a brine. The heating of a brine, at a temperature equal to or upper than $100^{\circ} \mathrm{C}$, during 45 to $60 \mathrm{mn}$, allows, in the presence of calcium carbonate and calcium sulphate germs, to proceed simultaneously to the sterilization and to the chemical purification of brines.

\section{Ubersicht}

Die, den Käselaken zugebrachten chemischen Unreinigkeiten, haben drei Ursprunge : das Salz, das Auflösungswasser, die Käse.

Weiterhin, stellt man eine bakterische und Pilzsaurehaltige Bereicherung beim Altern der Salzlaken, fest.

Um diese Hindernisse zu vermeiden hat man ein klassisches Pasteurisieren vorgenommen, d.h. eine Ultraviolettstrah'en Behandlung, oder ein Pasteurisieren durch Hinzufügung antiseptischen Produkten.

Keines dieser Verfahren hat bis jetzt erlaubt ganz zufriedenstellende Ergebnisse zu erhalten und eine konjugierte Sterilisierung und chemische Reinigung Behandlung der Salzlake war bis jetzt nicht möglich. Das Heizen der Salzlake bei einer Temperatur von $100^{\circ} \mathrm{C}$ oder mehr, während 45 bis 60 Minuten, erlaubt ein gleichzeitiges Sterilisieren und chemische Reiningung der Salzlaken, bei Gegenwart von Kalkcarbonat und Kalksulfat, ohne Inkrustieren der Heizungsanlagen.

\section{Bibliographie}

[1] Casalis (J.), Luquet (F. M.) et Rossier (F.) (1969), - Sur le traitement des saumures de fromagerie par les rayons ultra-violets. Le Lait, 49, 134-145.

[2] Centeleghe (J. L.), Milliere (J. B.), Veillet (L.) et Weber (F.) (1971). Aspects physico-chimiques et microbiologiques du salage en saumure des pâtes molles moisies. La Technique Laitière, sous presse.

[3] Centeleghe (J. L.), Milliere (J. B.), Veillet (L.) et Weber (F.) (1971). Etude de survies de quelques souches bactériennes dans les saumures. Travaux de l'Ecole Supérieure de Laiterie de Nancy, non encore publiés.

[4] Futschiк (J.) (1961). - Uber die Entkeimung von Salzbädern. Ost. Milchw., 16, 233-234.

[5] Hinterberger (S.) (1964). - Salzbad und seine Entkeimung. Ost. Milchw., 19, 401-404.

[6] Lacrampe (J. L.), Hardy (J.) Ramet (J. P.) et Weber (F.) (1971). - Contribution à l'étude de l'évolution chimique et du traitement des saumures de fromagerie. Le Lait, $L I, 158-175$.

[7] Viard (M.) (1964). - A propos du salage en saumure des pâtes molles. Techicien du lait, 197, 5-14. 